\title{
Hacia un Mercado Único Digital: Caso práctico del Proyecto europeo: Promoting the AS4 between PEPPOL Access Points around EU
}

\author{
Hermenegildo Gil-Gómez ${ }^{\mathrm{a}}$, Doina Stratu-Strelet ${ }^{\mathrm{b}}$, José Antonio-Lozano ${ }^{\mathrm{c}}$, Vicente \\ Guerola-Navarro ${ }^{\text {d. }}$ \\ ${ }^{\mathrm{a}-\mathrm{d}}$ Departamento de Organización de Empesas, Universitat Politècnica de València. Valencia
}

\begin{abstract}
Resumen
La Unión Europea está eliminando las barreras transfronterizas en las transacciones comerciales dentro del territorio europeo, fomentando así la economía digital. La estrategia del Mercado Único Digital busca que los ciudadanos europeos puedan acceder libremente a bienes y servicios en línea.
\end{abstract}

En esta comunicación se presentará la estrategia del Mercado Único Digital $y$ se analizará como caso de estudio el proyecto 'Promoting the AS4EU between PEPPOL Access points around EU-AS4EU' financiado por la Comisión Europea dentro de su programa CEF Telecom.

Palabras clave: Intercambio electrónico de datos, AS4EU, Mercado Único Digital

\section{Marco del Proyecto}

\subsection{El Mercado Único Digital}

Las tecnologías de la información y las comunicaciones se están convirtiendo en la base de los sistemas económicos modernos. Estos cambios están ocurriendo a una velocidad que ofrece muchas oportunidades para la innovación, el crecimiento sostenible y el empleo. La Unión Europea establece que un Mercado Único Digital es aquel en el que se garantiza la libre circulación de bienes, personas y servicios permitiendo a las empresas y ciudadanos acceder a las transacciones en línea en condiciones de competencia leal, y un alto nivel de protección de datos (EURLEX,2019). 
Internet y las tecnologías de la información están cambiando el campo de la gestión, pero las barreras que existen pueden disminuir las oportunidades de negocio propio a un mercado integrado. El Mercado Único Digital tiene como objetivo reducir las barreras y ofrecer más oportunidades para realizar transacciones en línea de una forma segura y asequible en toda la Unión Europea, protegiendo al consumidor y fomentando la economía digital. Solo el 7\% de las pequeñas y medianas empresas venden en el extranjero, pero esto puede aumentar con la implementación plena de la etapa de integración digital, en especial con la estrategia del Mercado Único Europeo (European Commission, 2019).

Según (European Commsision, 2019) el Mercado Único Digital tiene los siguientes objetivos:

- Impulsar el comercio electrónico en la Unión Europea eliminando el bloqueo geográfico, haciendo que la entrega de bienes y servicios transfronterizos sea más eficiente.

- Modernizar las normas de derechos de autor de la Unión Europea para adaptarse a la era digital, abordando el contenido digital en línea y protegiendo a los usuarios más vulnerables.

- Intensificar la respuesta de Europa a los ciberataques, creando una disuasión cibernética y de derecho penal de la Unión Europea, protegiendo así a los ciudadanos, empresas e instituciones públicas,

- Apostar por el potencial la economía de datos, facilitando su flujo e intercambio por todo el territorio europeo. Garantizando a su vez la protección de los datos personales

- Garantizar que todos los Estados Miembros de la Unión Europea tenga una buena conexión a Internet.

- Ayudar a las empresas, investigadores, ciudadanos y autoridades a emplear las herramientas digitales, garantizando que todos tienen las habilidades para ello.

En conclusión, la estrategia del Mercado Único Digital consiste en transformar la sociedad europea en base a la ola de digitalización que se está produciendo en todo el mundo.

\section{2. eDelivery Building Block}

Denominamos eDelivery Building Block a una red de nodos para intercambio electrónico de datos a nivel europeo. Se basa en un modelo distribuido en el que cada punto de acceso se convierte en un nodo utilizando protocolos de transporte y políticas de seguridad estándar. El eDelivery eDelivery Building Blocl apoya a las administraciones públicas a 
intercambiar datos y documentos electrónicos con otras administraciones públicas, empresas y ciudadanos, de manera interoperable, segura, confiable. Está por componentes basados en especificaciones, software y servicios reutilizables que formarán parte de una amplia variedad de sistemas de IT en diferentes dominios de políticas de la UE.

El componente básico CEF eDelivery se basa en el protocolo de mensajería AS4, abierto y gratuito para todos, desarrollado por la organización de desarrollo de estándares OASIS. Para facilitar su adopción en Europa, eDelivery utiliza las pautas de implementación de AS4 definidas por los Estados miembros en e-SENS Large Scale Pilot. Las organizaciones deben instalar un punto de acceso o utilizar un proveedor de servicios para intercambiar información con el protocolo de mensajería AS4 (CEF Digital, 2019).

El eDelivery Building Block está en relación directa con el Reglamento eIDAS UE 910/2014. En sus conclusiones el Consejo Europeo invitó a la Comisión Europea a contribuir al Mercado Único Digital mediante la creación de condiciones apropiadas para el reconocimiento mutuo entre los Estados Miembros, de herramientas electrónicas, tales como identificación electrónica, documentos electrónicos, firmas electrónicas y servicios de entrega electrónica, y para servicios interoperables de gobierno electrónico en toda la Unión Europea. De igual forma, el Reglamento eIDAS dio un paso muy importante, estableciendo que los datos enviados y recibidos utilizando un servicio de entrega registrado electrónico tendrán efectos legales y la admisibilidad como prueba en procedimientos judiciales (EURLEX, 2019).

Por lo tanto, el objetivo de CEF eDelivery es que aquellas administraciones públicas que hayan desarrollado su sistema IT de forma independiente puedan intercambiar cualquier tipo de documentos y datos de forma segura con otras administraciones públicas, empresas y ciudadanos (European Commision, 2019d).

\subsection{Beneficios del Intercambio Electrónico de Datos}

Según (CEF Digital, 2019) las características del intercambio electrónico de datos, con los consiguientes beneficios que se ofrece al sector público, privado y al ciudadano, se pueden resumir de la siguiente forma:

1. Una de las características es la interoperabilidad, esto es, el intercambio electrónico de datos está basado en especificaciones técnicas comunes que permiten a diversas organizaciones intercambiar datos y documentos. Esto se traduce en los siguientes beneficios:

- Se permite el intercambio de documentos y / o datos utilizando protocolos de mensajería estandarizados que no sean correo electrónico. 
- Se permite el intercambio de archivos adjuntos además de mensajes (documentos XML)

- $\quad$ Se permite visualizar fácilmente la dirección registrada de los nodos

- $\quad$ Se permite ejecutar interacciones asíncronas de solicitud-respuesta.

2. La segunda característica es la seguridad. Los beneficios son:

- Los participantes están seguros de que los datos y documentos están protegidos contra cualquier modificación (integridad)

- Están seguros de que los documentos se cifran durante la transmisión (confidencialidad)

- Están seguros de que el origen y el destino de los datos y documentos son confiables

- Tiene acceso a registros avanzados y configurables de eventos relacionados con el intercambio de datos y documentos.

3. La característica de escalabilidad y rendimiento, Permite que crezca el número de participantes en la red de intercambio de datos, así como el número de mensajes intercambiados.

4. Puede intercambiar documentos y archivos de datos de más de $50 \mathrm{Mb}$.

5. Garantía legal y responsabilidad: se promueve un alto nivel de transparencia y confianza entre todos los participantes en la red de intercambio de mensajes.

- Se tiene la garantía de que los datos y documentos se entregan una vez y solo una vez (reintentos, recibos, eliminación de duplicados)

- Se está seguro de que los mensajes se entregan

\section{El Proyecto AS4EU}

El proyecto "Promoting the AS4 between PEPPOL Access Points around EU (AS4EU)" se encaja dentro de los proyectos CEF eDelivery. El proyecto AS4EU fue coordinado por la Universitat Politècnica de Valencia (UPV). En este proyecto, el consorcio AS4EU introducirá el protocolo AS4 en las soluciones de los EDI Providers participantes y desarrollará el SMP de acuerdo con la Plataforma de Servicios Centrales promovida por CEF Digital. En esta ocasión, el proyecto se presentó en forma de proyecto competitivo, y fue financiado por Comisión Europea a través de su instrumento de financiación 
denominado Connecting Europe Facility (CEF Telecom, 2019), en especial, a través de la convocatoria denominada CEF Telecom- eDelivery 2017-2 (CEF-TC-2017-2).

Para llevar a cabo el proyecto, el consorcio está formado por cuatro proveedores de servicios electrónicos (SATA, Celtrino, DataInterchange y AdValvas) cuyo objetivo es convertirse en un Punto de Acceso gracias a la implementación del protocolo de comunicación AS4 que ofrece en abierto los eDelivery Building Blocks. Se pretende que con esta implementación se permitirá que los cuatro proveedores de servicios electrónicos puedan ofrecer al mercado un servicio de intercambio de documentos electrónicos más seguro y que opera a mayor escala, conectando administraciones públicas, empresas y ciudadanos. Este proyecto promueve el intercambio de datos electrónicos de forma segura y de acuerdo con los estándares europeos, cumpliendo con el Reglamento (UE) n 910/2014.

\subsection{Objetivos}

El objetivo principal de este proyecto es promover el uso del servicio DSI eDelivery en entidades públicas y privadas, implementando cuatro Puntos de Acceso e integrando el protocolo AS4 y los correspondientes SMP (Service Metadata Publisher).

\subsection{Participantes en el proyecto}

1. Universitat Politècnica de València (UPV), España (Institución Coordinadora)

2. Leading Management Technology S.L. (LMT), España

3. S.A.T.A Applicazione Tecnologie Avanzate Srl (S.A.T.A), Italia

4. EDI FACTORY LTD (Celtrino), Irlanda

5. DATA INTERCHANGE PLC (Interchange), Reino Unido

6. AdValvas Europe NV (AdValvas), Bélgica

\subsection{Fases del proyecto}

Las fases en las que se desarrolló el proyecto fueron las siguientes:

- Gestión administrativa y financiera del proyecto: los objetivos de esta actividad fue coordinar al consorcio, monitorizar el progreso del proyecto y la monitorizar que se conseguían los objetivos establecidos en el Grant Agreement. Dentro de esta actividad se incluyeron tareas de gestión financiera y administrativa para monitorizar los costes del proyecto y el cumplimiento de las relaciones contractuales con la entidad otorgante de la subvención, en este caso la Comisión Europea; tareas de gestión del conocimiento adquirido durante el periodo de implementación de los puntos de acceso; y tareas de 
ejecución de auditorías internas garantizando el cumplimiento de los estándares de calidad establecidos en la propuesta original del proyecto.

- Análisis de requisitos técnicos: en esta actividad se desarrollaron tareas preparatorias para la implementación técnica, se incluyeron tareas de análisis de los requerimientos técnicos y tareas de creación de una estrategia de implementación.

- Implementación técnica e integración del protocolo de comunicación AS4 en SATA, Celtrino, DataInterchange, y AdValvas: esta actividad incluye tareas de configuración del protocolo de comunicación; tareas de implementación del protocolo y conexión con el SMP; y tareas relativas a las pruebas de interoperabilidad y conformidad.

- $\quad$ Comunicación y difusión: como parte del contrato de subvención se establece la obligación de difundir la financiación europea para el proyecto subvencionado a nivel nacional y europeo.

\subsection{Principales resultados}

Los resultadnos alcanzados por el proyecto AS4EU ha generado un impacto positivo en las entidades implementadoras, en sus clientes B2B y B2G en particular, y en el mercado europeo en general. Los resultados alcanzados han sido:

1. La implementación exitosa de los estándares de CEF eDelivery Building Block en las plataformas cloud de los 4 proveedores tecnológicos, convirtiéndose en puntos de acceso AS4 interoperables entre ellos y con otras organizaciones. Una vez finalizado el proyecto distintas organizaciones podrán intercambiar datos y documentos de forma segura utilizando un protocolo de mensajería estandarizado en toda la Unión Europea.

2. La eliminación de barreras en las transacciones comerciales en la Unión Europea, promoviendo la integración económica y el Mercado Único digital.

3. La integración de SATA, Celtrino, DataInterchange y AdValvas en la red CEF eDelivery, convirtiéndose en proveedores de un servicio de confianza: un servicio de entrega electrónica certificada que garantiza la seguridad y la integridad de los datos a transmitir (los datos están protegidos contra cualquier modificación), la confidencialidad del mensaje, y la confiabilidad del origen y el destino de los datos.

4. Los datos que se intercambien a través de los nuevos servicios de entrega electrónica certificada tendrán el mismo efecto jurídico y probatorio que un documento en papel, ya que, disfrutarán de la presunción de la integridad de los datos, de que el envío de dichos datos se ha realizado por el remitente identificado, y la recepción por el destinatario identificado y la exactitud de la fecha y hora de envío y recepción de los datos. 
5. Reducción de los costes de transacción y los errores administrativos en las cadenas de suministro de las organizaciones conectadas a los puntos de acceso (Gutiérrez, 2016). Esto aumentará la productividad, la eficiencia y la escalabilidad de las organizaciones, haciendo crecer sus beneficios gracias a la tecnología y convirtiéndose en organizaciones más competitivas dentro del mercado europeo.

\section{Conclusiones}

Con este proyecto se ha demostrado que los proveedores de servicios electrónicos están apostando por las infraestructuras digitales europeas y el mercado único digital, siendo la financiación europea un elemento motivador clave. Las políticas europeas están consiguiendo movilizar a distintos agentes económicos, que se unen en consorcios públicoprivados con el objetivo de gestionar eficazmente la innovación y el desarrollo tecnológico.

Desde el punto de vista de los resultados del proyecto, la estandarización técnica es la vía más eficaz para establecer una ruta óptima hacia la interoperabilidad de los diversos agentes económicos. El mantenimiento de un único estándar en el intercambio electrónico de datos facilita el avance hacia una mayor seguridad y eficiencia en los procesos económicos, burocráticos y digitales. Los CEF Building Blocks actúan como una base y pilar para promover un crecimiento paralelo de los distintos proveedores de tecnología, permitiéndoles mejorar su propia plataforma en un ambiente interconectado.

\section{Agradecimientos}

Los autores agradecen a la Comisión Europea la financión al Proyecto "Promoting the AS4 between PEPPOL Access Points around EU (AS4EU)" (Ref.: INEA/CEF/ICT/A2017/1527217 Action number: 2017-EU-IA-0104) dentro del programa "Connecting Europe Facility (CEF).

\section{Referencias}

CEF Telecom, 2019. Consultado octubre 2019. Disponible en: https://ec.europa.eu/inea/en/connecting-europe-facility/cef-telecom/apply-funding/2019-ceftelecom-calls-proposals

European Commission, 2019. Digital Single Market. Consultado octubre 2019. Disponible en: https://ec.europa.eu/commission/priorities/digital-single-market_es 
Hacia un Mercado Único Digital: Caso práctico del Proyecto europeo: Promoting the AS4 between PEPPOL Access Points around EU

EURLEX, 2019. Comunicación de la Comisión al Parlamento Europeo, al Consejo, al Comité Económico y Social y al Comité de las Regiones. Consultado ocutbre 2019. Disponible en: https://eur-lex.europa.eu/legal-content/ES/TXT/?uri=CELEX\%3A52018DC0029

European Commission, 2019. CEF Digital. Consultado octubre 2019. Disponible en:https://ec.europa.eu/cefdigital/wiki/display/CEFDIGITAL/Documentation+eDelivery

EURLEX, 2019. Reglamento (UE) no 910/2014 del Parlamento Europeo y del Consejo, de 23 de julio de 2014, sobre identificación electrónica y servicios de confianza para transacciones electrónicas en el mercado interior y por el que se deroga la Directiva 1999/93 / CE. Consultado en octubre 2019. Disponible en: https://eur-lex.europa.eu/legalcontent/EN/TXT/?uri=uriserv:OJ.L_.2014.257.01.0073.01.ENG

European Commission (2010). Communication from the Commission to the European parliament, the Council, the European Economic and Social Committee and the Committee of Regions: A Digital Agenda for Europe, COM (250)245

European Commission (2012). Artículo 26 del Tratado de Funcionamiento de la Unión Europea 2012/C 326/01

European Commission (2017a). Estrategia para el mercado único digital en la Unión Europea. Comunicación de la Comisión al Consejo, al Comité Económico y Social y al Comité de las Regiones (COM/2017/0228, FINAL,10-5-2017)

European Commission. (2017b). CEF Telecom Call - eDelivery (CEF-TC-2017-2). From CEF Telecom. URL: https://ec.europa.eu/inea/en/connecting-europe-facility/cef-telecom/applyfunding/2017-cef-telecom-call-edelivery-cef-tc-2017-2

European Commission (2019a). New EU rules on e-commerce from DIGITAL SINGLE MARKET. URL: https://ec.europa.eu/digital-single-market/en/new-eu-rules-e-commerce

European Commission. (2019b). The Vision of a Connected Europe. From CEF DIGITAL. URL: https://ec.europa.eu/cefdigital/wiki/display/CEFDIGITAL/The+Vision

European Commission. (2019c). What is eDelivery. From CEF DIGITAL. URL: https://ec.europa.eu/cefdigital/wiki/display/CEFDIGITAL/What+is+eDelivery

European Commission. (2019d). CEF Glossary. From CEF DIGITAL. URL: https://ec.europa.eu/cefdigital/wiki/display/CEFDIGITAL/CEF+Glossary

European Commission. (2019e). CEF Glossary. From CEF Telecom. URL: https://ec.europa.eu/inea/sites/inea/files/annex_cid_cef-tc-2017-2.pdf

European Parliament (2012). Regulation (EU) No 1025/2012 of the European Parliament and of the Council of 25 October 2012 on European standardisation, amending Council Directives 
89/686/EEC and 93/15/EEC and Directives 94/9/EC, 94/25/EC, 95/16/EC, 97/23/EC, 98/34/EC, 2004/22/EC, 2007/23/EC, 2009/23/EC and 2009/105/EC of the European Parliament and of the Council and repealing Council Decision 87/95/EEC and Decision No 1673/2006/EC of the European Parliament and of the Council Text with EEA relevance

European Parliament (2014). REGLAMENTO (UE) No 910/2014 DEL PARLAMENTO EUROPEO Y DEL CONSEJO de 23 de julio de 2014 relativo a la identificación electrónica y los servicios de confianza para las transacciones electrónicas en el mercado interior y por la que se deroga la Directiva 1999/93/CE. 